\title{
External validity of phase III trials on vaccines against SARS-CoV-2 to a middle-aged and elderly Western European population
}

\author{
Natalie Terzikhan ${ }^{1} \cdot$ Albert Hofman $^{2} \cdot$ Jaap Goudsmit ${ }^{2,3,4} \cdot$ Mohammad Arfan Ikram $^{1}$
}

Received: 8 December 2020 / Accepted: 8 February 2021 / Published online: 26 February 2021

(c) The Author(s) 2021

\begin{abstract}
Initial results from various phase-III trials on vaccines against SARS-CoV-2 are promising. For proper translation of these results to clinical guidelines, it is essential to determine how well the general population is reflected in the study populations of these trials. This study was conducted among 7162 participants (age-range: 51-106 years; 58\% women) from the Rotterdam Study. We quantified the proportion of participants that would be eligible for the nine ongoing phase-III trials. We further quantified the eligibility among participants at high risk to develop severe COVID-19. Since many trials were not explicit in their exclusion criterion with respect to 'acute' or 'unstable preexisting' diseases, we performed two analyses. First, we included all participants irrespective of this criterion. Second, we excluded persons with acute or 'unstable preexisting' diseases. $97 \%$ of 7162 participants was eligible for any trial with eligibility for separate trials ranging between 11-97\%. For high-risk individuals the corresponding numbers were $96 \%$ for any trial with separate trials ranging from 5-96\%. Importantly, considering persons ineligible due to 'acute' or 'unstable pre-existing' disease drastically dropped the eligibilities for all trials below $43 \%$ for the total population and below $36 \%$ for high-risk individuals. The eligibility for ongoing vaccine trials against SARS-CoV-2 can reduce by half depending on interpretation and application of a single unspecified exclusion criterion. This exclusion criterion in our study would especially affect the elderly and those with pre-existing morbidities. These findings thus indicate the difficulty as well as importance of developing clinical recommendations for vaccination and applying these to the appropriate target populations. This becomes especially paramount considering the fact that many countries worldwide have initiated their vaccination programs by first targeting the elderly and most vulnerable persons.
\end{abstract}

Keywords SARS-CoV-2 $\cdot$ Vaccines $\cdot$ Phase III trials $\cdot$ Generalizability $\cdot$ External validity $\cdot$ Epidemiology

\section{Introduction}

Several phase III trials on vaccines against SARS-CoV-2 are ongoing and initial results highly promising. A major issue of these phase III trials is to what extent the included

Mohammad Arfan Ikram

m.a.ikram@erasmusmc.nl

1 Department of Epidemiology, Erasmus University Medical Center, Wytemaweg 80, 3015 CN Rotterdam, the Netherlands

2 Department of Epidemiology, Harvard T.H. Chan School of Public Health, Boston, MA, USA

3 Human Immunomics Initiative, Department of Epidemiology, Harvard T.H. Chan School of Public Health and Human Vaccines Project, Boston, MA, USA

4 Department of Immunology and Infectious Diseases, Harvard T.H. Chan School of Public Health, Boston, MA, USA study population is representative of the intended (or target) population, i.e. external validity.

For these trials, the intended target population is initially comprised of high-risk individuals usually considered to be elderly persons as well as those with comorbidities, and ultimately the entire world population. It remains unclear whether these target populations are representatively recruited into ongoing trials. This information is pivotal, since clinical recommendations for any approved vaccine should incorporate the proper target populations for which these vaccines have shown efficacy, and also determine those populations not sufficiently represented in the trials.

We sought to quantify the external validity of the various ongoing trials to a middle-aged and elderly West-European population from the Rotterdam Study. Specifically, we were interested to quantify what proportion of this study population would be eligible to participate in these trials and how many of those eligible are from high-risk categories. 


\section{Methods}

Extensive methods are available in the Online Resource.

Briefly, for this study we screened www.clinicaltrials.gov for ongoing phase III trials focused on vaccine development against SARS-CoV-2 and COVID-19.

The group at high risk of severe COVID-19 was defined according to the criteria of the Dutch National Institute for Public Health and the Environment (RIVM) [1], and included participants aged 70 or higher, and participants with asthma and COPD, diabetes, cancer, participants with current use of antineoplastic and immunosuppressive agents, obesity, end-stage kidney disease, liver steatosis and cirrhosis and cardiac diseases.

We carried out our analyses in the population-based Rotterdam Study [2] and used data collected from 2009 to 2014, which yielded 7162 persons (mean age 70 years (SD 9.8), $58 \%$ women) for analysis (Online Resource Table 1). These calendar-years were chosen such to maximize the number of living participants as well as their available data. Data on the comorbidities was ascertained during the in-person examinations complemented by automated linkage of medical and pharmacy records to our study database.

We applied eligibility criteria from each separate trial to our study population and calculated the following proportions: the proportion of participants eligible for any trial, and for each trial separately, the number of high-risk individuals in our study eligible for any trial, and for each trial separately.

We performed two complementary analyses and calculated the abovementioned proportions in each analysis

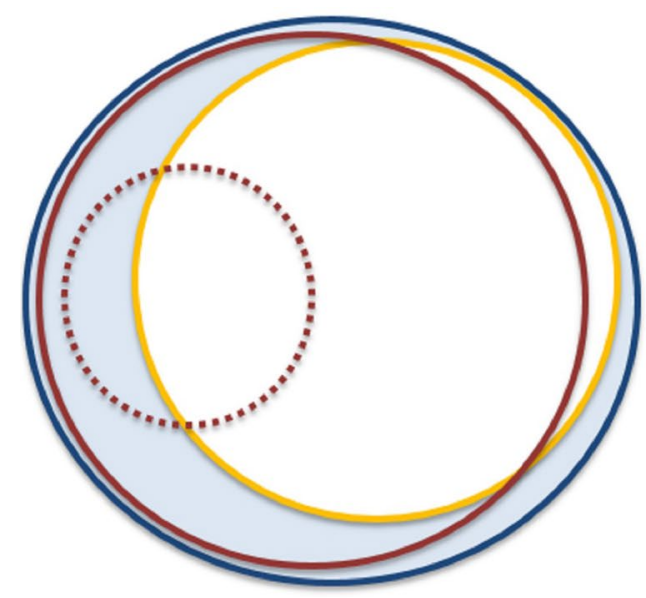

Fig. 1 Venn diagram for the proportion of eligible Rotterdam Study participants for any trial. The colours are coded as follows: blue circle: total study population; yellow circle: persons at high risk of severe COVID-19; red circle: persons eligible for any trial in the first separately. These two analyses differed with respect to the interpretation of an eligibility criterion that was not always explicitly specified in the various trial protocols. This criterion was often stated as follows: 'preexisting (un)stable disease', 'an acute course of disease', or 'other medical or psychiatric condition or laboratory abnormality that may increase the risk of study participation or, in the investigator's judgment, make the participant inappropriate for the study'. In our dataset, we operationalized this criterion as follows: diagnosis of dementia, diagnosis of moderate to severe COPD, current clinically significant depressive symptoms, abnormal kidney function, current liver disease (defined as liver steatosis, and liver cirrhosis) or a new diagnosis within the preceding three months for the following conditions: stroke, cancer, (including antineoplastic agents), diabetes mellitus, COPD, cardiac disease (heart failure, myocardial infarct, atrial fibrillation, and revascularisation). In the first analysis, we included everyone as eligible, who met this operationalization and in the second analysis, we excluded anyone who met this operationalization. The general characteristics of individuals with or without acute or unstable disease are presented in Online Resource Tables 2 and 3).

Finally, in sensitivity analyses we incrementally restricted the study population to persons aged over 60 , 70, and 80 years (Online Resource Fig. 1).

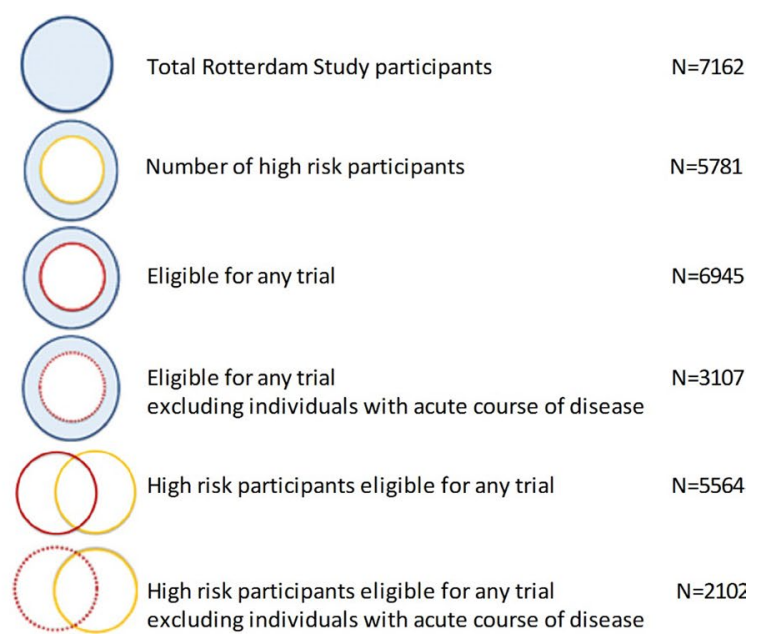

analysis; red dotted circle: persons eligible for any trial in the second analysis, in which individuals with acute course of disease were excluded 


\section{Results}

Table 1 presents the exclusion criteria from the 9 included trials, and we note that seven of these mentioned the eligibility criterion of 'acute' or 'unstable preexisting' disease without further specification. Therefore, we performed two analyses to calculate the proportion of the eligible participants for each trial. In the first analysis, we included all eligible participants, including those who met our operationalization of the eligibility criterion of 'acute' or 'unstable preexisting' in the seven trials that mentioned this criterion without further specification. In the second analysis, we repeated the first analysis but now excluding those who met our operationalization of 'acute' or 'unstable preexisting' criterion.

97\% ( $\mathrm{N}=6945)$ of the total Rotterdam Study participants would be eligible for any trial in the first analysis, while this percentage dropped to $43 \%(\mathrm{~N}=3107)$ in the second analysis. Among the 5781 participants at high-risk of severe COVID-19, $96 \%(\mathrm{~N}=5564)$ would be eligible for any trial in the first analysis and $36 \%(\mathrm{~N}=2102)$ in the second analysis (Fig. 1).

Figure 2a shows the percentages for the two analyses for each trial separately. Whereas in the first analysis the proportion included for the most inclusive trial was $97 \%$, this number dropped considerably in the second analysis to $43 \%$. Figure $2 \mathrm{~b}$ shows the corresponding numbers from participants at high-risk of severe COVID-19. Finally, sensitivity analyses revealed similar patterns at various age cut-offs (Online Resource Fig. 1).

\section{Discussion}

In a middle-aged and elderly population in the Netherlands from predominantly West-European descent, we found that $97 \%$ of this population would be eligible to participate in any of the nine currently ongoing vaccine trials against SARS-CoV-2. For persons at high-risk of severe COVID19 , the eligibility for any trial was $96 \%$. Importantly, applying stricter exclusion based on the criterion 'acute' or 'unstable preexisting' disease drastically reduced the eligibility for any trial to $43 \%$ of the entire study population and $36 \%$ of the high-risk individuals.

Clinical trials are often considered the golden standard in efficacy research, due to several strengths by design with respect to internal validity. In contrast, whether findings from clinical trials are adequately translated to clinical practice also depends on their external validity. External validity is the extent to which findings from one study are applicable to target populations not represented in the actual study population. To properly gauge the actual study population, it is crucial that clinical trials explicitly report the setting of the trial, the exact intervention, the inclusion and exclusion criteria, and the characteristics of the actually recruited population $[3,4]$ in [5]. For the ongoing clinical trials, any effective vaccine can be considered effective in similar settings as the original trial. The judgement whether that vaccine will be also effective in other settings, i.e. external validity, is based on prior knowledge, biological plausibility, statistical considerations and eligibility criteria of the original trial [3]. Many countries worldwide have prioritized the elderly and those most vulnerable in their vaccination campaigns, indicating that policy-makers consider the external validity of the ongoing trials to these populations sufficient.

The population of the Rotterdam Study is a lower-middle class population of primarily European descent. Previously, this population has shown good generalizability to the population of the Netherlands [6-8]. Another important metric in this regard is the response rate, which has continuously exceeded $70 \%$ for the Rotterdam Study [2]. This is a major strength of our study which makes it not only populationbased but also population-representative, and far exceeds response rates for other larger efforts ongoing worldwide [9]. Notwithstanding these considerations, the drastic drop in eligibility for the stricter criterion in the second analysis would likely have been of the same magnitude in any other population, irrespective of geographical or ethnic setting or response rate.

In conclusion, we found that eligibility for ongoing vaccine trials against SARS-CoV-2 can reduce by half depending on interpretation and application of a single unspecified exclusion criterion. This exclusion criterion in our study would especially affect the elderly and those with pre-existing morbidities. These findings thus indicate the difficulty as well as importance of developing clinical recommendations for vaccination and applying these to the appropriate target populations. This becomes especially paramount considering the fact that many countries worldwide have initiated their vaccination programs by first targeting the elderly and most vulnerable persons.

Supplementary Information The online version contains supplementary material available at https://doi.org/10.1007/s10654-021-00729-5.

Author contributions $\mathrm{AH}, \mathrm{JG}$ and MAI conceptualized the analysis. NT and MAI did the data analysis and prepared the first draft of the manuscript. All authors were involved in the interpretation of the results and revision of the final manuscript.

Funding The Rotterdam Study is funded by Erasmus Medical Center and Erasmus University, Rotterdam, Netherlands Organization for the Health Research and Development (ZonMw), the Research Institute for Diseases in the Elderly (RIDE), the Ministry of Education, Culture and Science, the Ministry for Health, Welfare and Sports, the European 
Table 1 Overview of the included trials with the selection of exclusion criteria per trial, for which data from the Rotterdam Study was available

Trial sponsor (collaborator) Clinical Trials.gov identifier

\section{ModernaTX, Inc \\ (Biomedical advanced research and develop- ment authority and NIAID)}

NCT04470427

Immunodeficient state and therapy*

Acute or unstable disease, not further specified

\section{Janssen vaccines and prevention B.V}

NCT04505722

Immunodeficient state and therapy*
Butantan Institute

(Sinovac Life Sciences Co., Ltd.)

\section{NCT04456595}

Immunodeficient state and therapy*

Acute or unstable disease, not further specified

Alcohol dependency

Depressive symptoms

Dementia diagnosis

Acute or unstable disease, not further specified

AstraZeneca

(Iqvia Pty Ltd)

NCT04516746

Immunodeficient state and therapy*

History of malignancy ${ }^{b}$

Acute or unstable disease, not further specified

Gamaleya Research Institute \& Health

Ministry of the Russian Federation

(Government of the city of Moscow and CRO)

\section{NCT04530396}

Immunodeficient state and therapy*

History of neoplasms

Alcohol dependency

Acute stroke the previous year

Acute cardiac disease in the previous year

Acute or unstable disease, not further specified

\section{BioNTech SE}

(Pfizer)

\section{NCT04368728}

Immunodeficient state and therapy*

Age $>85$ years

Dementia diagnosis

Depression

Acute or unstable disease, not further specified

\section{China National Biotec Group Company Limited}

(G42 Healthcare company, Abu Dhabi Health Services Company, Wuhan Institute of Biological Products Co., Ltd and Beijing Institute of Biological Products Co., Ltd) NCT04510207

Immunodeficient state and therapy*

DiaBP $>90 \mathrm{mmHg}$

SysBP $>150 \mathrm{mmHg}$

Dementia diagnosis

Acute or unstable disease, as specified in the footnote $^{\mathrm{e}}$
Novavax

\section{NCT04583995}

Immunodeficient state and therapy*

Current diagnosis/treatment for cancer

Alcohol dependency

Continuous use of anticoagulants ${ }^{\mathrm{d}}$

History of chronic neurological disorders that have required prior specialist medical review ${ }^{\mathrm{a}}$

Age $>84$ years

Acute or unstable disease, not further specified NPO Petrovax

(CanSino Biologics Inc.)

\section{NCT04540419}

Immunodeficient state and therapy*

History of malignancies ${ }^{\mathrm{c}}$

Age $>85$ years

History of diabetes mellitus

$18.5<\mathrm{BMI}>30$

SysBP $>139 \mathrm{mmHg}$

DiaBP $>90 \mathrm{mmHg}$

SysBP $<100 \mathrm{mmHg}$

DiaBP $<60 \mathrm{mmHg}$

Acute or unstable disease, as specified in the footnote

$B M I$ body mass index, DiaBP diastolic blood pressure, SysBP systolic blood pressure

*Immunosuppressive and immunomodulatory medications: Immunosuppressive medications, corticosteroid use and antineoplastic agents; ICD10-codes: L04, L01, H01

${ }^{a}$ History of chronic neurological disorders that have required prior specialist medical review: Dementia, Parkinson Disease, Stroke in the previous year

${ }^{\mathrm{b}}$ Except childhood cancers and prostate cancer and uterine cervical carcinoma

${ }^{\mathrm{c}}$ Including history of lymphoma and history of haematopoietic cancer

${ }^{\mathrm{d}} \mathrm{ICD} 10$ - codes: B01AA and B01AE

${ }^{\mathrm{e}}$ Acute or unstable disease is defined as: Dementia diagnosis, diagnosis of moderate to severe Chronic obstructive pulmonary disease (COPD), current depressive symptoms, abnormal kidney function (defined as estimated Glomerular Filtration Rate $<60$ millilitre/minute), current liver disease (defined as liver steatosis, and liver cirrhosis). Diagnosis of the following within the previous 3 months: stroke, cancer (including antineoplastic agents), diabetes mellitus, COPD, cardiac disease (heart failure, myocardial infarct, atrial fibrillation, and revascularisation) 
Total population $(\mathrm{N}=7162)$

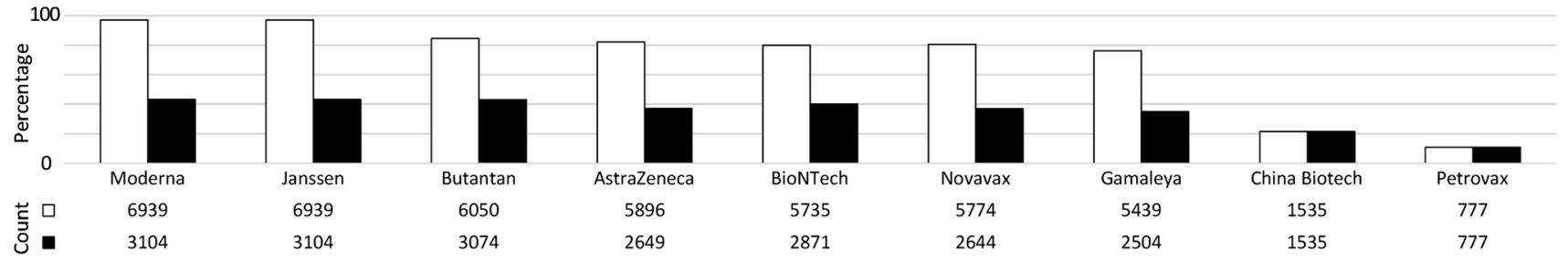

Population at high risk for COVID-19 ( $N=5781)$

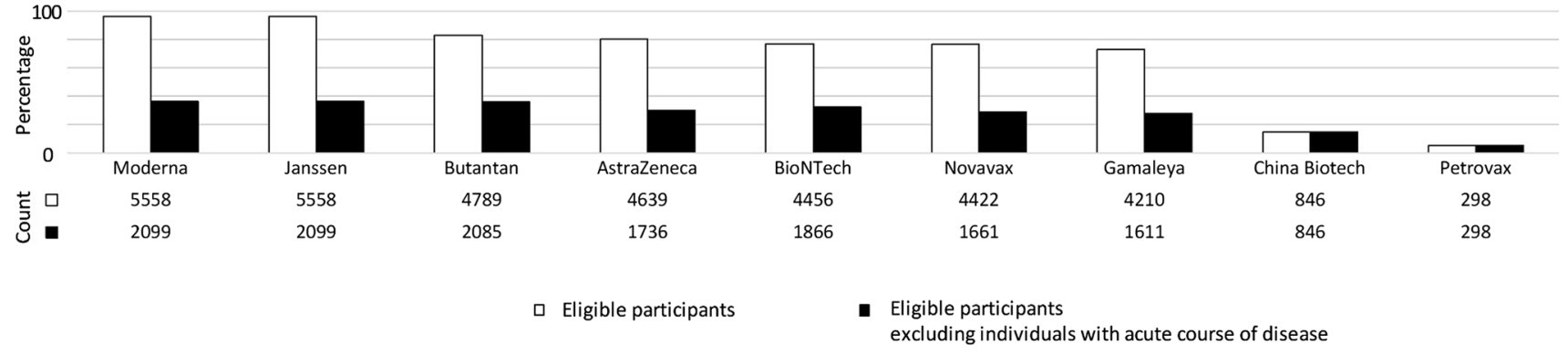

Fig. 2 The number and proportion of eligibility from the Rotterdam Study population for ongoing clinical phase III trials on a vaccine against SARS-CoV-2. White bars indicate data from the first analysis; black bars indicate data from the second analysis. The difference between these two analyses is the operationalization, and thus inclu-

Commission (DG XII), and the Municipality of Rotterdam. The authors are grateful to the study participants, the staff from the Rotterdam Study and the participating general practitioners and pharmacists.

Availability of data and material Data can be obtained upon request. Requests should be directed towards the management team of the Rotterdam Study (secretariat.epi @erasmusmc.nl), which has a protocol for approving data requests. Because of restrictions based on privacy regulations and informed consent of the participants, data cannot be made freely available in a public repository.

\section{Compliance with ethical standards}

Conflict of interest Authors declare no conflicts of interests.

Ethical approval The Rotterdam Study has been approved by the Medical Ethics Committee of the Erasmus MC (registration number MEC 02.1015) and by the Dutch Ministry of Health, Welfare and Sport (Population Screening Act WBO, license number 1071272-159521PG). The Rotterdam Study Personal Registration Data collection is filed with the Erasmus MC Data Protection Officer under registration number EMC1712001. The Rotterdam Study has been entered into the Netherlands National Trial Register (NTR; www.trialregister.nl) and into the WHO International Clinical Trials Registry Platform (ICTRP; www.who.int/ictrp/network/primary/en/) under shared catalogue number NTR6831. All participants provided written informed consent to participate in the study and to have their information obtained from treating physicians. sion or exclusion, of the eligibility criteria '(un)stable disease', 'acute course of disease', or 'other condition increasing risk of participation'. a total population of the Rotterdam Study and $\mathbf{b}$ individuals at high risk of severe COVID-19

Open Access This article is licensed under a Creative Commons Attribution 4.0 International License, which permits use, sharing, adaptation, distribution and reproduction in any medium or format, as long as you give appropriate credit to the original author(s) and the source, provide a link to the Creative Commons licence, and indicate if changes were made. The images or other third party material in this article are included in the article's Creative Commons licence, unless indicated otherwise in a credit line to the material. If material is not included in the article's Creative Commons licence and your intended use is not permitted by statutory regulation or exceeds the permitted use, you will need to obtain permission directly from the copyright holder. To view a copy of this licence, visit http://creativecommons.org/licenses/by/4.0/.

\section{References}

1. RIVM. Risicogroepen en COVID-19. 2020 10-11-2020]; Available from: https://www.rivm.nl/coronavirus-covid-19/risicogroe pen.

2. Ikram MA, et al. The rotterdam study: 2018 update on objectives, design and main results. Eur $\mathrm{J}$ Epidemiol. 2017;32(9):807-50.

3. Dekkers OM, et al. How to assess the external validity of therapeutic trials: a conceptual approach. Int J Epidemiol. 2010;39(1):89-94.

4. Pibouleau L, et al. Applicability and generalisability of published results of randomised controlled trials and 
non-randomised studies evaluating four orthopaedic procedures: methodological systematic review. BMJ. 2009;339:b4538.

5. Braend AM, Straand J, Klovning A. Clinical drug trials in general practice: how well are external validity issues reported? Bmc Fam Pract. 2017;18:1-11.

6. Licher S, et al. Lifetime risk and multimorbidity of non-communicable diseases and disease-free life expectancy in the general population: a population-based cohort study. PLoS Med. 2019;16(2):e1002741.

7. Vernooij MW, et al. Incidental findings on brain MRI in the general population. N Engl J Med. 2007;357(18):1821-8.

8. Vektis. Factsheet Chronische aandoeningen bij 40-plussers. 2019; Available from: https://www.vektis.nl/intelligence/publi caties/factsheet-chronische-aandoeningen-bij-40-plussers.
9. Winkler V, et al. Response in individuals with and without foreign background and application to the National Cohort in Germany: which factors have an effect? Int J Public Health. 2014;59(3):555-63.

Publisher's Note Springer Nature remains neutral with regard to jurisdictional claims in published maps and institutional affiliations. 